\title{
EDUCACIÓN AMBIENTAL PARA EL USO EFICIENTE DEL AGUA POTABLE EN ALUMNOS DE QUINTO DE PRIMARIA DE TRES INSTITUCIONES EDUCATIVAS EN LA CIUDAD DE TACNA
}

ENVIRONMENTAL EDUCATION FOR THE EFFICIENT USE OF DRINKING WATER IN FIFTH GRADERS FROM TREE EDUCATIONAL INSTITUTIONS IN THE CITY OF TACNA

María Briguitte Cori Tenorio ${ }^{1}$ Verónica del Rosario Maldonado Vásquez ${ }^{2}$

Marisol Mendoza Aquino ${ }^{3}$

Información del artículo:

Recibido: 20/09/2020

Aceptado: $15 / 11 / 2020$

\footnotetext{
${ }^{12}$ Escuela de ingeniería Ambiental, Universidad Privada de Tacna, Perú ${ }^{3}$ Docete escuela de ingeniería Ambiental, Universidad Privada de Tacna, Perú E-mail: ${ }^{1}$ mcoritenorio@gmail.com, ${ }^{2}$ vero95vk@gmail.com, ${ }^{3}$ mmendoza188@hotmail.com
} 


\section{Resumen}

La investigación tuvo como objetivo la elaboración de un programa educativo ambiental para concientizar a los alumnos de las tres instituciones educativas, sobre el uso eficiente del agua potable, mediante talleres informativos, actividades recreacionales para alumnos, docentes y padres de familia, se trabajó con 265 alumnos de quinto grado de primaria de las instituciones educativas; Coronel Bolognesi, República Argentina y Santa Cruz, antes de implementar el programa aplicó de un pre test de 30 preguntas con la finalidad de conocer el desempeño en el uso eficiente, reciclaje, optimización del agua. Posteriormente se aplicó un post test para, la data fue contrastada mediante la prueba estadística de signos. Los resultados indican que los bajos niveles de uso eficiente se redujeron de $36,6 \%$ a 1,0 \%, los niveles moderados se redujeron de 59 , $6 \%$ a $34,0 \%$, mientras que los niveles altos de eficiencia aumentaron de 3,8 \% a 65,0 \%. Respecto al reciclaje del agua, los valores que expresaban bajos niveles de eficiencia se redujeron de 32,1\% a $0,0 \%$, los niveles medios de $58,9 \%$ a $24,2 \%$ y los altos niveles de eficiencia de uso del agua aumentaron significativamente de $9,1 \%$ a $75,8 \%$, mientras que en la optimización del agua, los resultados encontrados evidencian que los niveles bajos se redujeron de 30,6 \% a 1,1\%, los niveles moderados de $66,4 \%$ a $35,5 \%$, favorablemente los niveles altos de eficiencia en el uso del agua aumentaron de $3,0 \%$ a 63,0 \%, todas las comparaciones fueron al $95 \%$ de confianza. Finalmente la institución educativa República Argentina destacó de manera sobresaliente en todos los aspectos evaluados con los niveles más altos, lo cual implica que la conciencia ambiental de sus estudiantes es adecuada.

Palabras claves: Agua potable; Educación ambiental; Eficiencia.

\section{Abstract}

The research aimed to develop an environmental educational program to raise awareness among the students of the three educational institutions, on the efficient use of drinking water, through information workshops, recreational activities for students, teachers and parents, 265 fifth graders from educational institutions were worked; Colonel Bolognesi, Argentina and Santa Cruz, before implementing the program applied a pre-test of 30 questions in order to know the performance in the efficient use, recycling, optimization of water. Subsequently a post test was applied for, the data were tested using the statistical significance test. Results indicate that low levels of efficient use were reduced from $36.6 \%$ to $1.0 \%$, moderate levels fell from $59.6 \%$ to $34.0 \%$, while high efficiency levels increased from $3.8 \%$ to $65.0 \%$. With regard to water recycling, values expressing low levels of efficiency were reduced from $32.1 \%$ to $0.0 \%$, average levels from $58.9 \%$ to $24.2 \%$ and high levels of water use efficiency increased significantly from $9.1 \%$ to $75.8 \%$, while in water optimization, found results show that low levels were reduced from $30.6 \%$ to $1.1 \%$, moderate levels of $66.4 \%$ to $35.5 \%$, favourably high levels of water use efficiency increased from $3.0 \%$ to $63.0 \%$, all comparisons were $95 \%$ confidence. Finally, the Argentine Republic educational institution excelled in all aspects evaluated at the highest levels, which implies that the environmental awareness of its students is adequate.

Keywords: Drinking water; Environmental education; Efficiency. 


\section{Introducción}

Varios autores han definido la educación ambiental (Berenguer de Santiago, 2020; De pinto, 2004; Acebal, 2010; Tornello, y Valladares, 2015) como los procesos que posibilitan la formación que permitan comprender la complejidad producida en el ambiente por la interacción de sus componentes naturales y socio-culturales que se resumen en actividades integradas en un proceso sistemático y permanente, dirigidas a promover cambios de comportamiento. En ese sentido, los procesos de educación deben ser permanentes, principalmente en aquellos donde la población adquiere formación académica, conciencia social y cultural, es importante que en todos los espacios se divulgue la forma consiente de actuar con el ambiente, en ese sentido el trabajo con estudiantes de primaria es una tarea irrenunciable con la consigna que educar a un niño es mejor que corregir los comportamientos del adulto. Por consiguiente el cuidado de algunos recursos escasos debe ser abordado a esos niveles, en particular en Tacna, ciudad enclaustrada en la cabecera del desierto de Atacama, se ha visto afectada por el problema hídrico, a causa del inadecuado uso y distribución que se da al recurso agua en actividades como ganadería, agricultura, piscicultura, gastronomía, según el ANA (2015), el 90\% se distribuye en el sector agrícola, sector minero $7 \%$, industria $0,1 \%$ y sector poblacional 3,7\%. Por consiguiente su uso apropiado es de vital importancia y como se mencionó, es sumamente importante a niveles de educación básica regular, al respecto se conoce que en el Perú, son muy pocos los centros educativos que se han involucrado de forma conjunta y voluntaria hacia un nuevo cambio, por intermedio de programas que realicen acciones de prevención y protección para el cuidado del ambiente, por falta de prácticas ambientales, hoy en día la mayoría de ciudades del Perú se ven afectadas por el problema de escasez hídrica o por contaminación de la misma, por inadecuados hábitos que han llevado a una situación crítica.

\section{Objetivos}

La investigación tiene como objetivo la aplicación de un programa de educación ambiental y concientización en los alumnos del quinto de primaria de dos instituciones educativos de Tacna.

\section{Metodología}

La investigación se desarrolló en seis etapas; la primera fue capacitar a los docentes de los centros educativos respecto a la problemática existente del agua y su recurso vital, también se presentó actividades para promover el uso eficiente del agua potable en los alumnos, la segunda etapa fue evidenciar la problemática y dar soluciones ante el mal uso del agua potable dentro de las instituciones educativas, como el desperdicio de agua en los baños, grifos que gotean, grifos abiertos, cisternas de agua en mal estado, entre otros, la tercera etapa consistió en la toma de un "pre test" conformado por 30 preguntas que fueron divididas en tres cuestionarios: uso eficiente del agua, reciclaje del agua y optimización del uso de agua, implementándose en el año 2019. Con la finalidad de diagnosticar el nivel 
de conocimientos de los alumnos de quinto grado de primaria, que se compone de 265 alumnos distribuidos en los tres centros educativos. La cuarta etapa fue concientizar a los alumnos sobre los malos hábitos que realizaban día a día en su centro educativo y en sus hogares, debido a las inadecuadas prácticas ambientales como regado de jardines, lavado de frutas o verduras, entre otros, pudiéndose reutilizar el agua. La quinta etapa se procedió a la realización de actividades recreacionales como fonomímicas, canto, marchas, charlas, etc., de esta manera se permitieron sensibilizar a los alumnos y padres con el fin de lograr un cambio en ellos y en la sexta etapa se tomó un "pos test", para comprobar si el programa tuvo un impacto significativo en los alumnos.

La investigación realizada tomó como referencia a Roldán (2016) quien realizó el estudio "Propuesta de optimización del uso del agua potable en la I.E. 80824 José Carlos Mariátegui el PorvenirTrujillo-2014" donde propone el uso de 3 encuestas a fin de poder conocer el nivel de educación de los alumnos y a las Guías Metodológicas, impartidas por la Superintendencia Nacional de Servicios de Saneamiento (SUNASS), guías aprobadas por el Ministerio de educación que comprende actividades orientadas hacia docentes, alumnos y padres de familia sobre el modo de insertar el tema del cuidado y ahorro del agua potable en las mallas curriculares de las instituciones educativas a nivel nacional y en sus hogares.

La población de estudio fueron alumnos de quinto grado de nivel primario, la I.E. Coronel Bolognesi con una cantidad de 96 alumnos con tres secciones (A, B y C), seguido de la I.E. República Argentina con 82 alumnos y la I.E. Santa Cruz con 87 alumnos, siendo cantidades representativas con un total de 265 alumnos, donde la edad promedio son de 10 a 12 años. Para el procesamiento de datos obtenidos mediante la aplicación de un test inicial y un test final se usó el programa IBM SPSS versión 25 y para el análisis de datos luego de la recolección de datos de los test se utilizó la prueba de signos.

Para la planificación de la investigación se realizó un programa de trabajo, que se resume de la siguiente manera:

a) Informando: sobre la problemática del agua actual.

b) Capacitando: ofreciendo la oportunidad ideal de ampliar o enriquecer conocimiento de alumnos, docentes y padres de familia

c) Sensibilizando: se procuró que la población alcance la capacidad de valorar el recurso agua.

d) Forjando Valores: como el respeto y la tolerancia, la que se adquiere mediante el trabajo en equipo y la convivencia a fin que los alumnos, los docentes y padres de familia puedan tomar mejores acciones para mitigar la problemática sobre el uso del agua en las I.E

Dicha planificación sirvió como herramienta fundamental en la organización de actividades bajo el concepto de "ciclo de Deming", para lograr la calidad de enseñanza y el mejoramiento continuo de acciones, con la finalidad de transformar las actividades de enseñanza-aprendizaje mediante actividades pedagógicas, adecuadas a las siguientes etapas:

- Planificar: en esta primera etapa consistió en involucrar planes de acción, para realizar un monitoreo en las instituciones educativas y de acuerdo a lo observado, realizar una tabla 
donde se "Jerarquiza los problemas ambientales" identificando criterios y alternativas de solución.

- Hacer: en esta etapa se procedió a aplicar el plan de educación identificando el mal uso del agua potable dentro de las I.E, procediendo así a trabajar con la comunidad educativa en general, y aplicando tres test para analizar el nivel de educación ambiental de los alumnos:

- Uso eficiente del agua potable: cuestionario que hace referencia si en I.E existían prácticas adecuadas respecto al uso correcto del agua potable.

- Reciclaje del agua potable: cuestionario que hace referencia si dentro de las I.E. los alumnos y docentes, incorporaban conocimientos o prácticas con respecto a una cultura de las "3Rs" con respecto al uso del agua potable.

- Optimización del agua: en este último cuestionario se consideraban la opción de reutilizar el agua potable para diversas actividades dentro de sus centros educativos u hogares.

Cada test impartido comprendía 10 interrogantes que ayudan a determinar el nivel de educación ambiental en los alumnos del quinto grado de primaria. Los resultados permitieron hacer el diagnostico general para planificar actividades, con un propósito definido que permitiese sensibilizar a los alumnos, docentes y padres de familia. Las actividades ayudaron a intensificar capacidades, conocimientos, actitudes y logros estas características personales.

- Verificar: la etapa de verificación de la mejoría deseada, se elaboró al finalizar el programa educativo y luego se procedió a la aplicación del Instrumento inicial a fin de conocer el impacto positivo del programa educativo y así comparar si los resultados muestran cambios significativo en los alumnos y según el centro educativo de procedencia.

- Actuar: la última etapa consistió en la estandarización, se diseñaron diversas herramientas que permitieron a los docentes participar en el Sexto programa educativo del ahorro del agua elaborado por SUNASS, donde los méritos obtenidos por las instituciones motivo de estudio, fueron óptimos.

Los programas educativos ambientales logran que los alumnos, docentes y padres de familia tomen conciencia, el valor de la protección de las fuentes de agua enfocado en el "Uso racional y responsable del agua, optimización del agua potable y el reciclaje del agua", actividades permitieron concientizar y mediante entrevistas y monitoreo permitió conocer las características personales frente al uso eficiente del agua potable en los alumnos de quinto de primaria de las I.E., finalmente cada centro educativo implementó un proyecto de innovación para el cuidado del agua, que integra a la comunidad estudiantil, promueve la equidad, trabajo en equipo, coherencia y desarrollo de proyectos, mejor calidad de vida agua segura. 


\section{Resultados}

\section{Uso eficiente del agua}

Luego de la ejecución de actividades educativas para sensibilizar a los alumnos de las instituciones educativas se procedió a tomar un pos - test que reflejan los resultados de la aplicación del programa de educación ambiental.

En la Figura 1 se presenta la distribución de frecuencias de tres niveles de Valoración (Bajo nivel, Moderado nivel y Alto nivel) tal como lo sugiere Roldán (2016), en el pre test de "Uso eficiente del agua", aplicado en los alumnos del quinto grado de primaria de las I.E. Coronel Bolognesi, República Argentina y Santa Cruz; con un total de 265 alumnos, resultó que el 36,6 \% obtuvieron un bajo nivel de eficiencia, seguidamente el 59,6\% con Moderado nivel eficiencia y por último el 3,8\% con alto nivel de eficiencia, mientras que en el post test el 1,0 \% obtuvieron un bajo nivel, seguidamente el $34 \%$ con moderado nivel y por último el 65,0 \% obtuvieron alto nivel. Según lo preciado existirían cambios significativos en el uso eficiente del agua, para comprobar estadísticamente se aplicó la prueba de signos basado en la prueba $Z$, donde resulto estadísticamente significativo ( $p$-Valor $=0,00$ ) al $95 \%$ de confianza.

\section{Figura 1}

Comparación de pre y post test del uso eficiente del agua
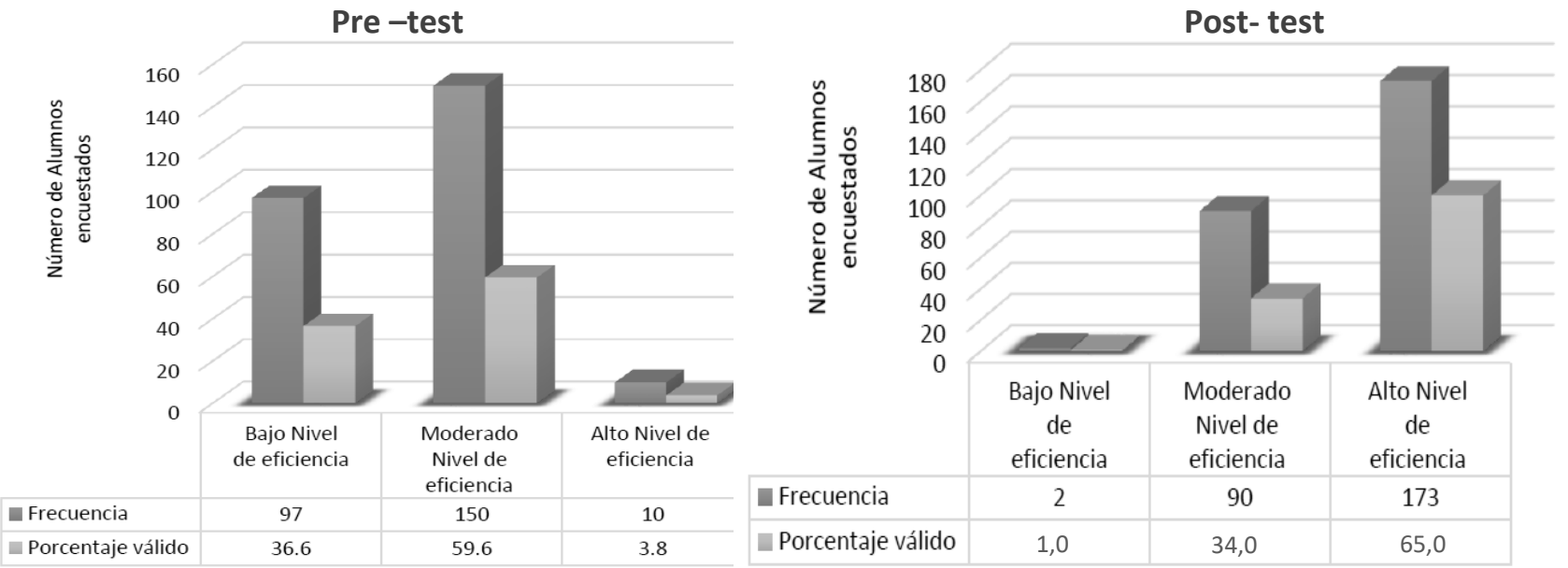

Nota. Elaboración propia.

En la figura 2 se observa el nivel alcanzado en el pos test sobre el "uso eficiente del agua" para las instituciones educativa, destacándose que la IE. República Argentina alcanzó los mayores niveles de eficiencia (84,10\%), seguido por la IE Santa cruz (60,90 \%) y Coronel Bolognesi $(53,10 \%)$. 


\section{Figura 2}

Representación del post test del uso eficiente del agua por instituciones educativas
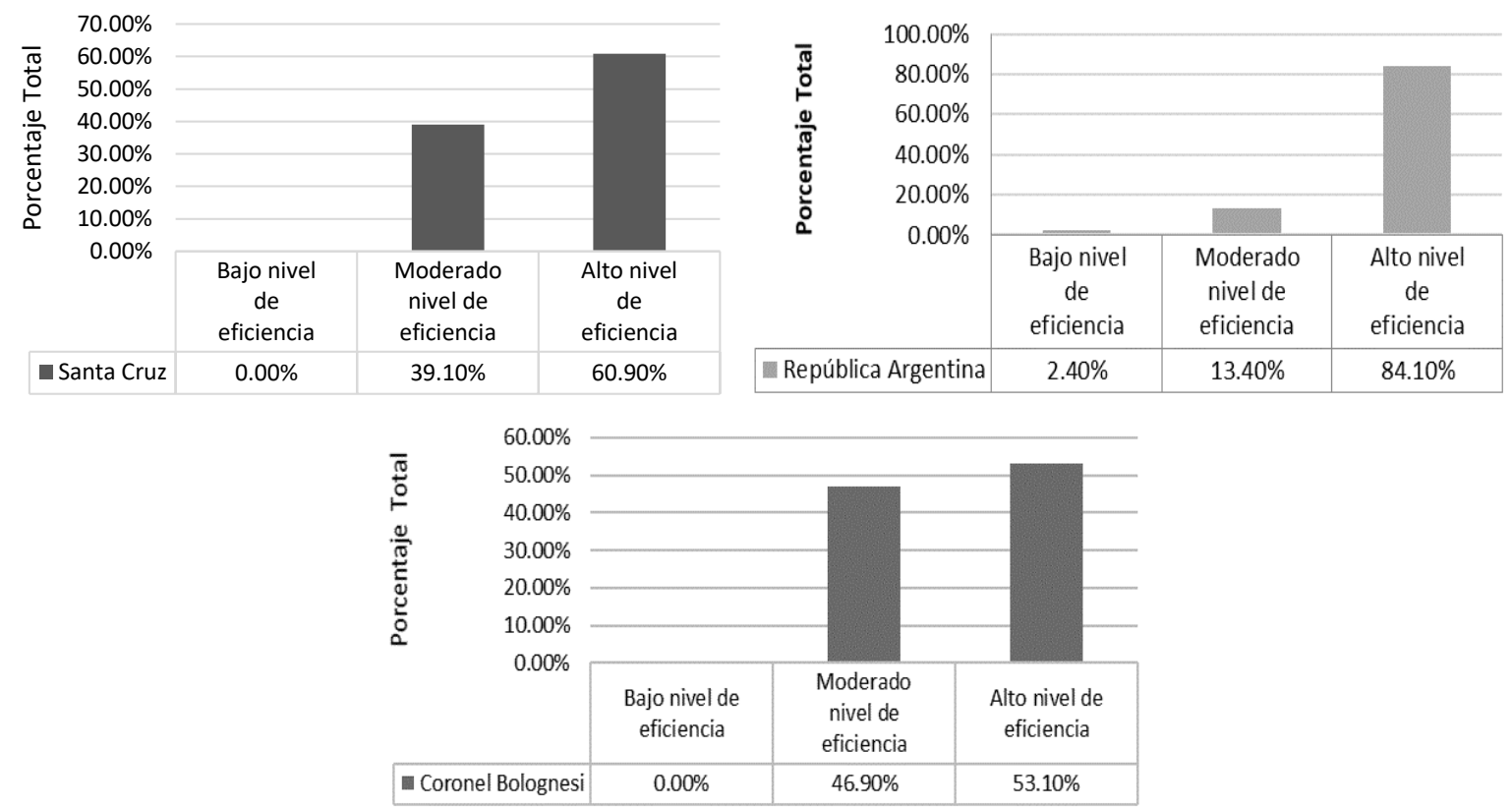

Nota. Elaboración propia.

\section{Reciclaje del agua}

En la Figura 3 se presenta la distribución en tres niveles de Valoración; Bajo nivel, Moderado nivel y Alto nivel del pre test referente al "Reciclaje del agua", resultando que el 32,1\% de alumnos mostraron tener un bajo nivel, seguido del 58,9 \% con moderado nivel y solo el 9,1 \% mostró tener altos niveles de reciclaje. En el post test $24,2 \%$ evidencio tener niveles moderados, destacándose que el $75,8 \%$ lograron un alto nivel de reciclaje del agua, así mismo que el nivel de eficiencia baja disminuyo a $0,0 \%$. En la comparación estadísticas entre el pre y post test existirían cambios significativos en el reciclaje del agua, para comprobar estadísticamente se aplicó la prueba de signos basado en la prueba Z, donde resulto estadísticamente significativo ( $p$-Valor $=0.00)$ al $95 \%$ de confianza, comprobándose que el programa resulto significativo y positivo.

En la figura 4 se observa el nivel alcanzado en el pos test sobre el "reciclaje del agua" para las instituciones educativa, destacándose que la IE. República Argentina alcanzó los mayores niveles de eficiencia $(86,60 \%)$, seguido por la IE Santa cruz $(85,10 \%)$ y Coronel Bolognesi $(58,30 \%)$. 
Figura 3

Comparación de pre y post test del Reciclaje del agua

Pre -test

200

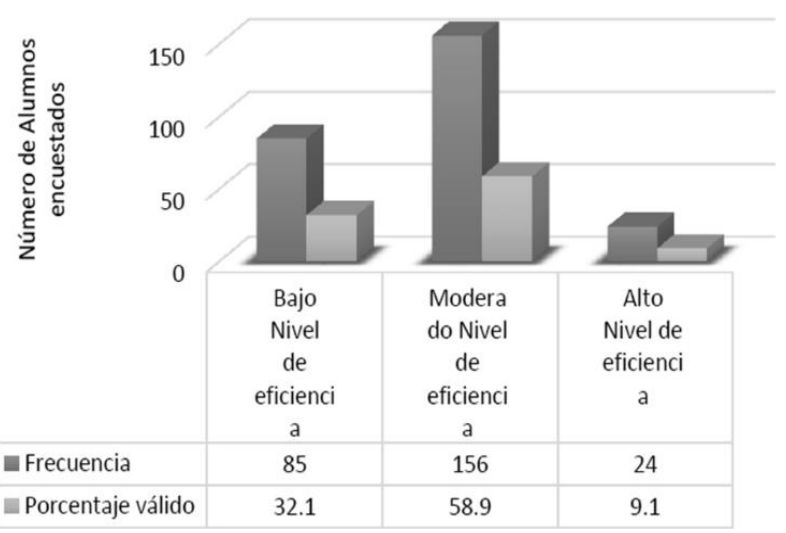

Post- test

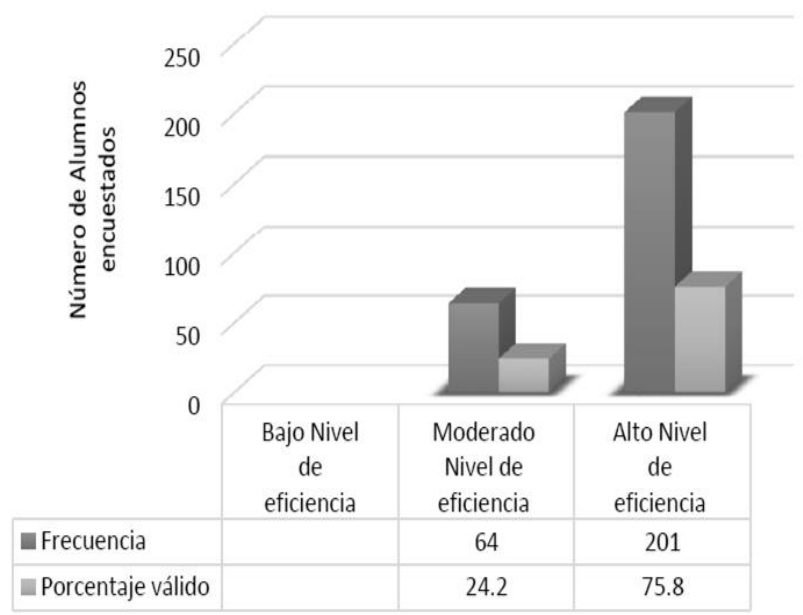

Nota. Elaboración propia.

Figura 4

Representación del post test del reciclaje del agua por instituciones educativas
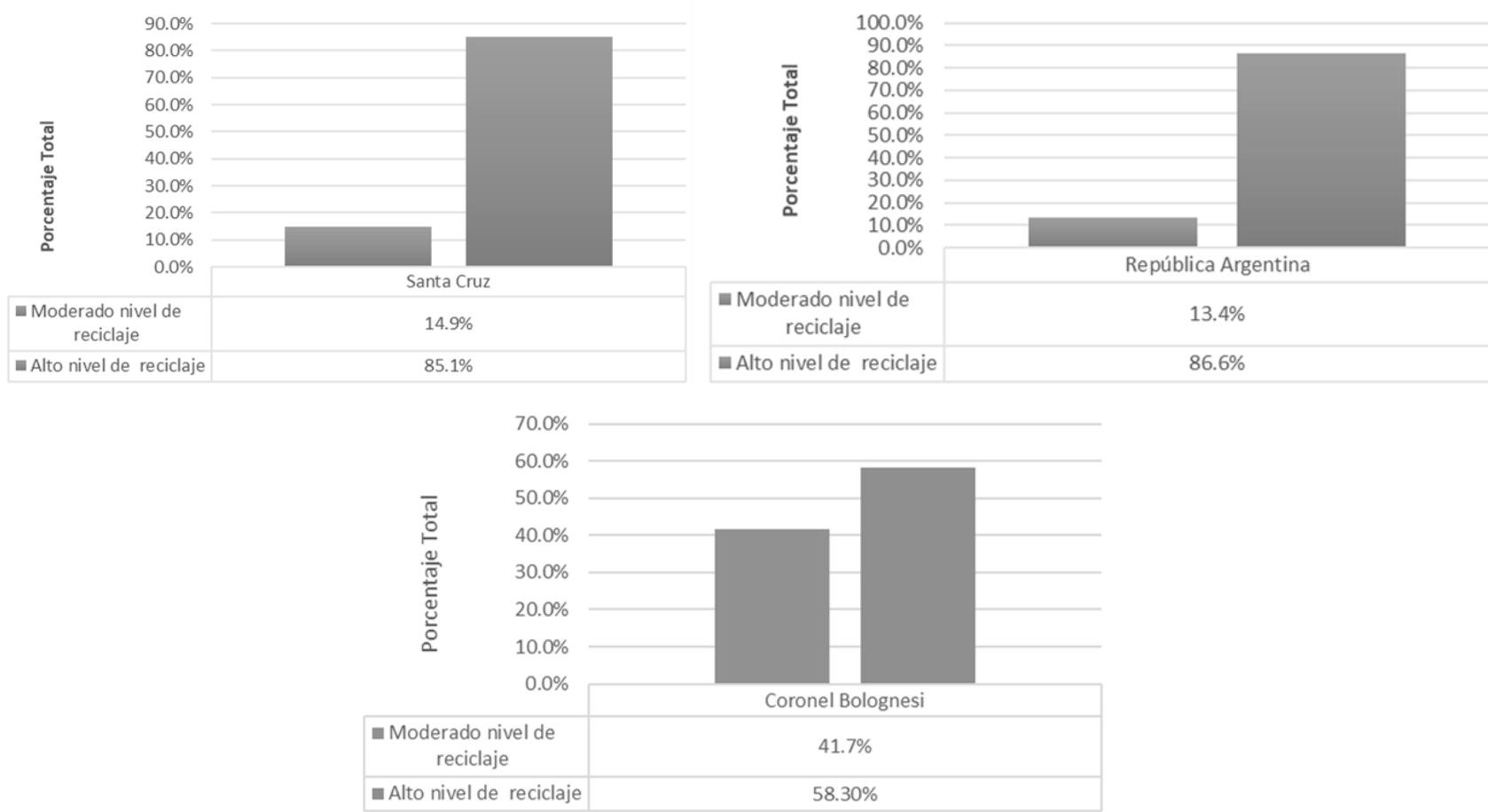

Nota. Elaboración propia. 


\section{Optimización del uso del agua}

En la Figura 5 se presenta la distribución de datos sobre el pre test de "Optimización del uso del agua", resultando que el 30,6 \% de alumnos mostraron bajo nivel de optimización del uso del agua, seguidamente el 66,4 \% moderado nivel mientras que un grupo minoritario de $3 \%$ de alumnos resultaron con altos niveles de optimización del uso de agua. Al aplicar el post test luego de implementar la capacitación, el bajo nivel de optimización del uso del agua se redujo a 1,1 \% mientras que los niveles moderados se redujeron a $35,8 \%$ y por el contrario los niveles altos subieron hasta el 63 $\%$, estos resultados muestran el estado final de conocimiento o percepción de cultura de agua en alumnos de quinto grado de primaria, según el ámbito de estudio.

Por otro lado, en la cotejo estadístico entre el pre y post test mostraron que existió cambios significativos en el reciclaje del agua, para comprobar estadísticamente se aplicó la prueba de signos basado en la prueba $Z$, donde resulto estadísticamente significativo ( $p$-Valor $=0.00$ ) al $95 \%$ de confianza, comprobándose que el programa sobre la optimización del uso del agua resultó significativo y positivo.

\section{Figura 5}

Comparación de pre y post test de la optimización del agua
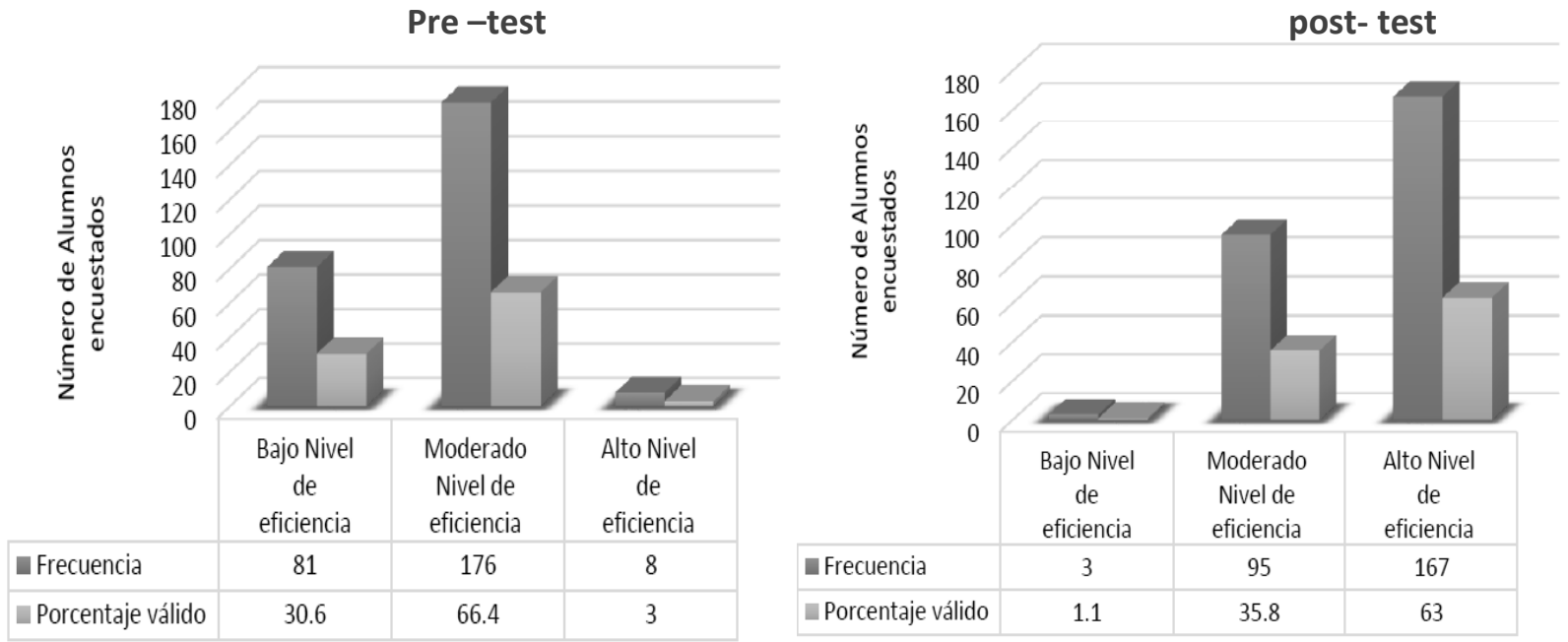

Nota. Elaboración propia.

Con la finalidad de evidenciar los niveles alcanzado en la optimización del agua por instituciones educativas, la figura 6 representa el post test por instituciones educativas, destacándose que la IE República Argentina, evidencia los niveles de optimización más altos (78\%), seguido de la IE Santa Cruz $(58,6 \%)$ y la IE Coronel Bolognesi $(54,2 \%)$, respecto a los niveles moderados destaca la IE Coronel Bolognesi $(43,8)$, seguido de la IE Santa cruz $(40,20 \%)$. La representación permite destacar que la IE República Argentina mostró niveles altos en todos los aspectos evaluados. 
Figura 6

Representación del post test de optimización del agua por instituciones educativas
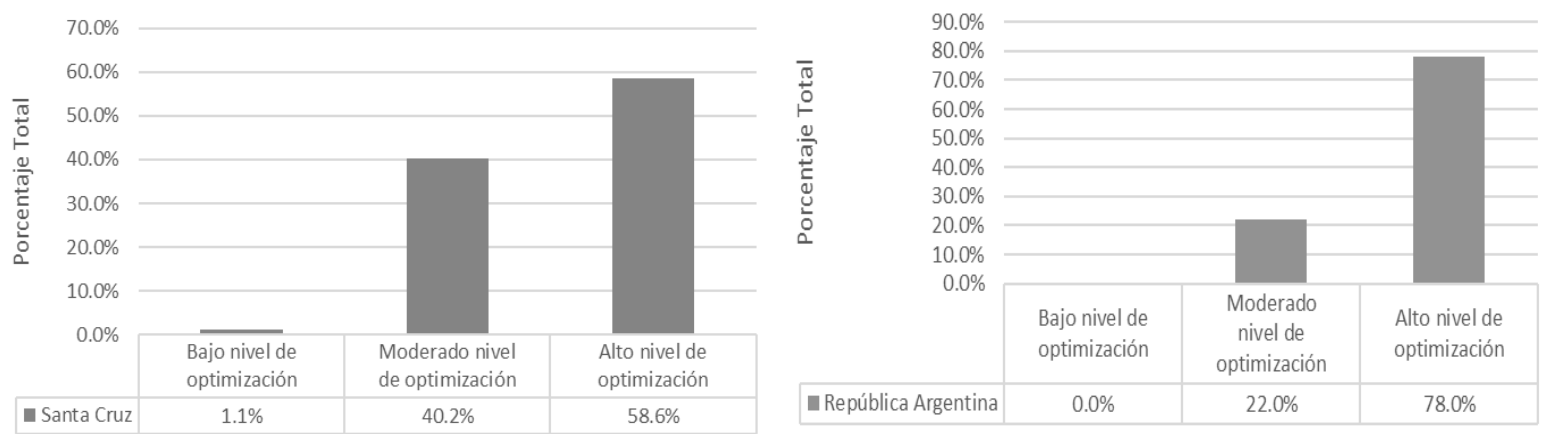

Nota. Elaboración propia.

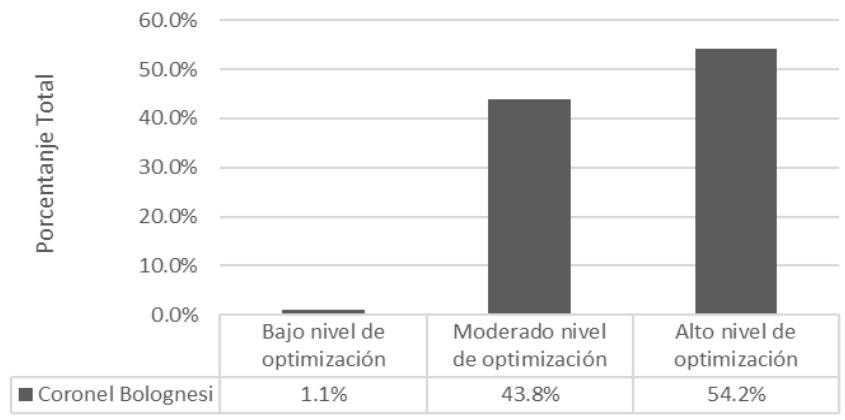

\section{Discusiones}

Según los resultados obtenidos, afirmamos que la hipótesis general sostiene que la educación ambiental si influye en los cambios de actitud sobre uso eficiente del agua potable en los alumnos de quinto de primaria de las I.E. Coronel Bolognesi, República Argentina y Santa Cruz, Tacna 2019, enfatizando la importancia de realizar acciones de sensibilización y concientización del uso del recurso agua. Los resultados obtenidos guardan similitud con Rodríguez (2016) e Yrene (2020) quienes sostienen que la Educación Ambiental es muy importante en la formación estudiantil porque busca promover valores y actitudes mediante diferentes proyectos ambientales en donde puedan conocer la importancia de la problemática hídrica y ambiental de su localidad. Resultados contrarios obtuvieron algunos autores como Angarita et al., (2018) al utilizar una metodología computarizada en los alumnos de quinto de primaria, que no mostraron interés alguno por estar pendientes de los móviles, por ello propone que antes de aplicar esta metodología es necesario evidenciar los componentes pedagógicos, tecnológicos, en la misma dirección Granados et al., (2015) sostienen que al haber aplicado un programa ambiental y encuestas, los alumnos del Institución educativa en las horas de receso, éstos juegan con el agua desperdiciándola con sus compañeros y los docentes al tener conocimiento de la problemática identificada, hicieron caso omiso a la ejecución de proyectos con solución ambiental.

En relación con la educación ambiental, los resultados son similares a lo encontrado por Chavesta (2018) quien logró mejorar el nivel de conocimientos en alumnos de primaria sobre el cuidado 
del agua con talleres de aprendizaje y aplicación de encuestas, además Matamoros et al., (2017) concluyen que mediante su programa de educación ambiental logró resultados satisfactorios en los pobladores de Callqui, donde aprendieron más del consumo de agua segura, también Orellana (2018) aplico el Plan Nacional de Educación Ambiental y comprobó en los alumnos de primaria una mejora en cuanto al desarrollo cognitivo y Roldán (2016) quien comparó los resultados antes y después de la aplicación de la propuesta de optimización de agua en escolares fueron favorables. Otros resultados discordantes son los obtenidos por Bucaram (2016) quien además de resaltar que las metodologías aplicadas son eficientes, explica que no generó cambio en los alumnos, concluyendo que el programa ambiental no tuvo éxito.

Respecto a la aplicación de un Programa de Educación Ambiental Fernández (2018) y Olivera (2019), demostraron que al aplicar un Programa de Educación Ambiental en los alumnos de primaria y secundaria, se consigue resultados satisfactorios respecto al nivel de conocimiento ambiental de los alumnos, mediante charlas de sensibilización, actividades recreativas con temática ambiental y estrategias ecológicas que permiten comprender a fondo la importancia del cuidado del agua y ambiente, también los resultados obtenidos por SUNASS (2018) en su Dirección de Educación, incorpora "La Cultura del Agua" en 1200 colegios de Tacna, , establecen que al aplicar un programa educativo ambiental a nivel local, las instituciones involucradas obtendrán resultados satisfactorios como lo hicieron años anteriores diversos centros educativos de la provincia de Tacna en el concursos nacional con temas de buenas prácticas para el cuidado del agua, enriqueciendo conocimientos ambientales en los alumnos y docentes.

\section{Conclusiones}

Se analizó el nivel de educación ambiental respecto al uso eficiente del agua potable en los alumnos de quinto de primaria de las I.E. Coronel Bolognesi, República Argentina y Santa Cruz, concluyéndose que en la dimensión uso eficiente del agua se redujo los bajos niveles de $36,6 \%$ a 1,0 \%, los niveles moderados se redujeron de 59, $6 \%$ a 34,0 \%, mientras que los niveles altos de eficiencia aumentaron de 3,8 \% a 65,0 \%, con una confianza estadística significativa al $95 \%$.

Respecto al Reciclaje del agua, los valores que expresan bajos niveles de eficiencia se redujeron de $32,1 \%$ a $0,0 \%$, los niveles medios de $58,9 \%$ a $24,2 \%$ y los altos niveles de eficiencia de uso del agua aumentaron significativamente de $9,1 \%$ a $75,8 \%$ con un nivel de confianza estadística del $95 \%$.

En la optimización del agua, los resultados encontrados evidencian que los niveles bajos se redujeron de $30,6 \%$ a $1,1 \%$, mientras que los niveles moderados se redujeron de $66,4 \%$ a $35,5 \%$, contrariamente los niveles altos de eficiencia en el uso del agua aumentaron de $3,0 \%$ a $63,0 \%$, con un nivel de confianza del $95 \%$.

Finalmente la institución educativa República Argentina destacó de manera sobresaliente en todos los aspectos evaluados con los niveles más altos en cada aspecto evaluado, lo cual implica que en dicha institución la conciencia ambiental de sus estudiantes es adecuada. 


\section{Referencias Bibliográficas}

Acebal Expósito, M. D. C. (2010). Conciencia ambiental y formación de maestras y maestros. Disponible en https://libros.metabiblioteca.org/handle/001/323

ANA. (2015). Plan de Gestión de los Recursos Hídricos de la Cuenca Caplina-Locumba. Lima: AUTORIDAD NACIONAL DEL AGUA. Obtenido de http://repositorio.ana.gob.pe/handle/20.500.12543/88

Angarita et al. (2018). Desarrollo de un MEC para la creación de cultura ciudadana sobre el uso del recurso hídrico en estudiantes de educación básica. Espacios, 39(15), 19. Obtenido de http://www.revistaespacios.com/a18v39n15/a18v39n15p19.pdf

Berenger de Santiago, B. (2002). La medida de las actitudes ambientales: propuesta de una escala de conciencia ambiental (Ecobarómetro). Psychosocial Intervention, 11(3), 349-358.

Bucaram. (2016). "Efecto de un programa de Educación Ambiental en la conducta de conservación de los recursos hídricos en estudiantes del Cantón Milagro, provincia del Guayas, Ecuador. UNIVERSIDAD NACIONAL DE TUMBES, TUMBES. Obtenido de http://repositorio.untumbes.edu.pe/handle/UNITUMBES/202

Chavesta. (2018). Conocimientos y Actitudes sobre el cuidado del ambiente en el recurso agua de los estudiantes del nivel secundario de la Institución Educativa Karl Weiss, Chiclayo 2017. UNIVERSIDAD DE LAMBAYEQUE, FACULTAD DE CIENCIAS DE INGENIERIA, CHICLAYO. Obtenido de https://repositorio.udl.edu.pe/xmlui/handle/UDL/115

De Pinto, E. P. (2004). Hacia una conciencia ambiental. Educere, 8(24), 34-40. Disponible en https://www.redalyc.org/pdf/356/35602406.pdf

Fernández. (2018). Educación Ambiental y la sensibilización en el manejo adecuado del Recurso Hídrico de los estudiantes del primer año de Secundaria del colegio 42021 Fortunato Zora Carbajal de la ciudad de Tacna en el año 2018. Universidad Privada de Tacna, Tacna. Obtenido de http://repositorio.upt.edu.pe/handle/UPT/1216

Granados et al. (2015). Diseño de un proyecto de ahorro y uso eficiente del agua, como estrategia que sensibiliza en el cuidado del recurso hídrico, a los estudiantes de la institución educativa Juan Pablo II del municipio de Palmira Valle. FUNDACIÓN UNIVERSITARIA LOS LIBERTADORES, Santiago Cali. Obtenido de

https://repository.libertadores.edu.co/bitstream/handle/11371/386/GranadosRamirezLorena.pdf ?sequence $=2 \&$ isAllowed $=\mathrm{y}$

Matamoros et al. (2017). Programa educativo "Agua segura" en el conocimiento sobre el consumo de agua en la comunidad de Callqui Chico, Huancavelica - 2017. Huancavelica. Obtenido de http://repositorio.unh.edu.pe/handle/UNH/1102

Olivera. (2019). Elaboración e implementación del proyecto de educación ambiental integrado Ecoinspirate en la Institución Educativa Miguel Pro. Tacna. Obtenido de http://repositorio.upt.edu.pe/handle/UPT/783

Orellana. (2018). Aplicación del plan nacional de educación ambiental en el desarrollo de la conciencia ambiental de los estudiantes de primaria en las escuelas ecoeficientes del distrito de San juan de Lurigancho UGEL $05 . \quad 151 . \quad$ Obtenido de http://repositorio.uigv.edu.pe/bitstream/handle/20.500.11818/3069/TESIS\%20DOCT.EDUC_GLOR 
IA\%20ERNESTINA\%20DE\%20LOS\%20R\%C3\%8DOS\%20ORELLANA\%20DE\%20FONTES.pdf?sequenc $\mathrm{e}=2$ \&isAllowed $=\mathrm{y}$

Rodríguez. (2016). La cultura del agua del organismo operador de agua potable, alcantarillado y saneamiento de Naucalpan de Juárez, (OAPAS). Universidad Nacional Autónoma de México, México. Obtenido de http://132.248.9.195/ptd2016/junio/0746721/Index.html

Roldán et al. (2016). Propuesta de optimización del uso del agua potable en la I.E. 80824 "José Carlos Mariátegui", El Porvenir Trujillo-2014. Trujillo. Obtenido de https://revistas.unitru.edu.pe/index.php/PGM/article/view/1158

SUNASS. (2014). Programa educativo: "Aprendiendo a usar responsablemente el agua potable". Obtenido de http://www.sunass.gob.pe/websunass/index.php/usuarios/concursos

SUNASS. (11 de abril de 2018). Dirección de Educación incorpora "La Cultura del Agua" en 1200 colegios de Tacna. Obtenido de http://www.sunass.gob.pe/websunass/index.php/noticias/noticiasregiones/item/1305-direccion-de-educacion-incorpora-la-cultura-del-agua-en-1200-colegios-detacna

Tonello, G., \& Valladares, N. (2015). Conciencia ambiental y conducta sustentable relacionada con el uso de energía para iluminación. Gestión y ambiente, 18(1), 45-59.

Yrene. (2020). Educación ambiental en el nivel media superior: estudio y acción en problemas ambientales. Universidad Nacional Autónoma de México, Morelia, Michoacán. Obtenido de file:///C:/Users/Usuario/Downloads/0799534.pdf 